\title{
Green neighbourhoods in low voltage networks: measuring impact of electric vehicles and photovoltaics on load profiles
}

\author{
Laura HATTAM ${ }^{\mathbf{1}}$, Danica Vukadinovic GREETHAM ${ }^{\mathbf{1}}$
}

\begin{abstract}
In the near future, various types of low-carbon technologies (LCTs) are expected to be widely employed throughout the United Kingdom. However, the effect that these technologies will have at a household level on the existing low voltage (LV) network is still an area of extensive research. We propose an agent based model that estimates the growth of LCTs within local neighbourhoods, where social influence is imposed. Real-life data from an LV network is used that comprises of many socially diverse neighbourhoods. Both electric vehicle uptake and the combined scenario of electric vehicle and photovoltaic adoption are investigated with this data. A probabilistic approach is outlined, which determines lower and upper bounds for the model response at every neighbourhood. This technique is used to assess the implications of modifying model assumptions and introducing new model features. Moreover, we discuss how the calculation of these bounds can inform future network planning decisions.
\end{abstract}

Keywords Agent based modelling, Low voltage networks, Electric vehicles, Photovoltaics

CrossCheck date: 18 November 2016

Received: 6 September 2016/Accepted: 18 November 2016/ Published online: 30 December 2016

(c) The Author(s) 2016. This article is published with open access at Springerlink.com

$\triangle$ Laura HATTAM

1.hattam@reading.ac.uk

Danica Vukadinovic GREETHAM

d.v.greetham@reading.ac.uk

1 Department of Mathematics and Statistics, University of Reading, Reading RG6 6AX, UK

\section{Introduction}

From 2005 onwards, the national electricity demand in the UK and other developed countries has stagnated or even decreased, despite the population increase. The UK energy statistics show that total electricity consumption year on year has reduced on a UK, GB and south-east level [1]. The current predictions are that the UK domestic electricity demand will continue to decrease in the next ten to fifteen years [2] due to the better efficiency of electrical appliances and lighting [3, 4]. The anticipated addition to the domestic demand will come mostly from the new builds and the low carbon technologies (LCTs) employed in existing buildings.

In particular, the electrification of transport and heating, which is forecasted for the near future, are predicted to be the main contributors to the changes in the electricity demand. The whole picture is made more complex by the variability of renewable energy sources, which result in new peaks and troughs forming in the aggregated consumption. However, not all the projected changes threaten to worsen this situation. A big mitigating factor is energy storage, which can help smoothen generation and demand, as well as offer cost efficient local solutions. Our proposed model can estimate future loads at the feeders, and as a result, street level storage solutions can be appropriately employed.

We are interested in measuring the combined impact of electric vehicles (EVs) and solar panels on low voltage (LV) networks. Several possible issues that might arise from the described smart grid developments are already recognised, these include frequent peak loads that reduce headway, voltage drops and phase unbalance. Due to the complexity of human societies, any predictions on the uptake of EVs and photovoltaics (PVs) comes with large 
uncertainties. Different models that determine uptake already exist and are used by network planners. However, by their nature it is quite difficult to validate these models, and to decouple the influence of different modelling parameters.

Our contribution is two-fold. Firstly, we present an agent based model of load profiles for the uptake of LCTs in local neighbourhoods when social influence is present. Our neighbourhoods are based on real-life LV networks containing multiple substations and feeders. This model uses a sample of realistic EV and PV profiles to simulate future uptake. Secondly, we demonstrate techniques that allow for a thorough mathematical analysis of results. Probabilistic methods based on multiple simulations enable the calculation of upper and lower bounds for the model response, which we refer to as confidence bounds. These bounds are used to understand the inner-workings of the model and to measure the effects of introducing/changing the model's parameters. In particular, these bounds are used here to quantify the difference between applying a clustered or random initial LCT distribution amongst our sample population.

The outline of the paper is the following. In Section 2 we give an overview of the recent relevant results. In Section 3 the model is described in detail, as well as the data that is used for the initialisation and calibration of the model. The focus is initially EV adoption only. In Section 4 the confidence bounds creation is explained with some simulation results shown. Then in Section 5 the adaptation of the model to include socio-demographic information is discussed. As well, confidence bounds are used to assess the impact of this new model feature. Next in Section 6 the model is further modified to investigate the combined uptake of EVs and PVs. Again, confidence bounds are computed to determine the effect of changing our model assumptions. Finally in Section 7 we discuss the implications of our results and their possible use in design, planning and policy.

\section{Previous work}

There is a fast growing amount of literature [5, 6] concerning the different impacts EVs and PVs will have on the future power grids. More specifically, these studies concentrate on load profiles, system losses, voltage profiles, phase unbalance, harmonic and stability impacts. Here, we focus on load profiles within a LV network.

\subsection{Impact of PVs and EVs to LV networks}

For EVs, most of the existing work is based on predictions, simulations or small pilot trials. Only recently have larger data sets based on trials become available. Focused mostly on LV networks impact, in [7] the authors created generic local networks to assess the neighbourhood impact of $\mathrm{EV}$ charging. Using a realistic distribution network simulation, in [8] the authors evaluate a range of different residential EV charging strategies, highlighting their strengths and weaknesses.

In [9], the authors aimed to measure the impact of PVs on LV networks in New Zealand. They were looking in particular at over-voltage and overload of conductors and transformers. They created a power-flow model of a $\mathrm{LV}$ network and simulated varying percentages of PV uptake. PV was based on a specific installation with an output power of $3.7 \mathrm{~kW}$. These uniform PVs were then randomly distributed through different parts of the LV network classified as rural, urban, industrial and city. Their results showed that only very high PV penetration (over 45\%) caused an overload of conductors, and in most cases overvoltage was not much higher than the existing statutory limit. In [5], the major technical impacts of small PV installations were discussed. This included excessive reverse power flow, overvoltages along distribution feeders, increased difficulty of voltage control, increased power losses (caused by reverse power flow) and severe phase unbalance.

A microgrid case-study from a neighbourhood in Utrecht in Netherlands, looking at the combination of PVs and EVs throughout a year was described in [10]. Based on simulations, and using February demand projected over the whole year, the authors compared several control algorithms. Their results showed a potential for relative peak reduction and increased self-consumption when using smart charging and vehicles to grid technology.

In [11], Monte-Carlo simulations were used to measure the impact of several low carbon technologies, including EVs and PVs. Similar to our approach, the authors used a realistic LV network with 7 feeders and sampled from realistic profiles for load and for LCTs. Note that the network area examined in our paper is significantly larger, with 44 feeders considered. Their focus is on identifying thermal and voltage problems in different feeders. While they use a random allocation of LCTs, we compare a random allocation with a clustered one using socio-demographic information, although here the focus is the load profile impact.

\subsection{Agent based modelling of PVs and EVs uptake}

In [12], a simple agent-based model of EV uptake was detailed and their impact on a local grid was examined. Their analysis was based on governmental scenarios of future UK EV uptake and a small pilot project that gave incentives to participants to charge overnight. As expected, 
having a variety of EV charging patterns helped to reduce the peaks, as opposed to when all the domestic charging occurred after work and overnight. By then comparing random and clustered uptake simulations, it was shown that some local grids might see a substantial increase of peak loads faster than expected. In [13], the impact of different EV charging behaviours on the electricity grid was as well considered, except their area of study was Western Australia. They concluded that by promoting off-peak charging, the effect of EV uptake on the grid would be minimised.

An agent-based model using San Francisco as a test city was presented in [14]. The paper considered how different policies and battery technologies might affect the uptake and usage of EVs. The model included a set of agents with socio-demographic properties, attitudes and an EV ecosystem that considered the cost of gas/electricity, rebates and public charging stations. Each three months, agents assessed whether they needed a new vehicle. Based on their properties, attitudes and state of their social network, they acquired (or not) an EV to use for their daily commute. The social network was created randomly based on similarities in age, income and residential locations of agents. This enabled the exploration of different scenarios (for example, increasing or decreasing rebates for EVs and increasing battery sizes) and then looking at the impacts on the average daily load.

\section{Agent based model}

The impact of future LCT adoption is predicted with our agent based model that applies a clustered distribution of technologies to a sample UK population (Bracknell, UK). The clustering follows the Joneses effect such that households are influenced by their neighbours' choices through observation, which is one of the causes for the development of 'green neighbourhoods'. This means a household is more likely to acquire a LCT if their neighbour already has one. Here, we model EVs and PVs as they are visible from the street, and can be observed by a neighbour.

Our network is based on a realistic LV network situated in Bracknell, UK, which comprises of 44 feeders. The household population at each feeder varies considerably. Figure 1 demonstrates the variation in feeder size, where the feeder number and feeder population is given. The feeders have been sorted according to feeder size. Note that each feeder corresponds to one neighbourhood and all households along a particular feeder are considered neighbours. Overall, there are 1841 properties, where 7 are households with PVs installed and 71 are commercial properties.

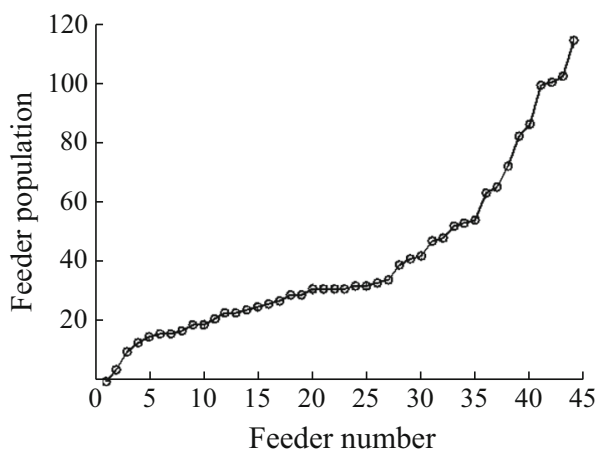

Fig. 1 Feeder populations

We have three data sets that were created using metered data from this LV network. This information was collected on Thursday the $15^{\text {th }}$ of January 2015 (winter), Thursday the $7^{\text {th }}$ of May 2015 (spring) and Thursday the $9^{\text {th }}$ of July 2015 (summer). The data sets consist of a combination of metered and predicted daily demand energy profiles $(\mathrm{kWh})$ for every household, where a genetic algorithm was used to allocate monitored endpoints to unmonitored customers [15]. These profiles have readings every half hour and therefore for each household we have a load profile as a vector of length 48 . Throughout this paper, we refer to these three data sets as 'baseloads'.

Thursdays are of particular interest here since presumably most household members will be at home and therefore, the demand will be maximised. As well, if they possess an EV, we assume Thursdays are a likely time to charge, just before the weekend.

Initially, the focus here is the clustered allocation of EVs, although later the combination of EV and PV uptake is investigated. The EV charging profiles used in our model were generated during the 55 week trial conducted by My Electric Avenue [16], where the number of participants increased as the trial progressed. These profiles consist of the two values ' 0 ' and ' 1.85 ' $\mathrm{kWh}$, which represent when the EV is not charging and charging respectively. They have readings also every half hour. Three days from this trial are selected, which are Thursday May the $8^{\text {th }} 2014$ (week 16 of the trial), Thursday July the $10^{\text {th }} 2014$ (week 25 of the trial) and Thursday January the $15^{\text {th }} 2015$ (week 52 of the trial). These dates are chosen since they correspond seasonally to the baseload dates. There are 79 households that consistently participate during weeks $16-52$ of the trial and therefore, we have $79 \mathrm{EV}$ daily profiles that are representative of winter, spring and summer charging behaviour. As an example, the EV profiles for one randomly selected household on the chosen days are shown in Fig. 2.

Note that EV profiles will have variability within each season. However, here we assume that by taking a snapshot of charging behaviour on Thursdays occurring at the same 


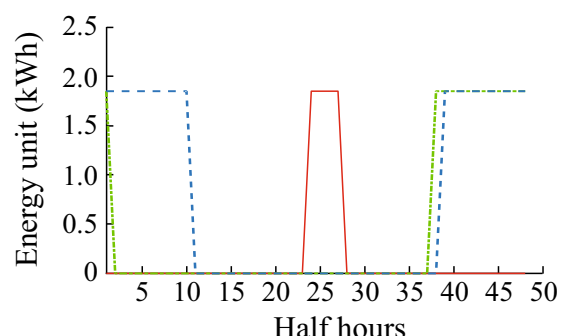

--- Week 16 (Spring); — Week 25 (Summer); - - - Week 52 (Winter)

Fig. 2 EV profiles for one household

time of year as the baseloads, the model result will be more realistic.

All profiles are depicted in energy units $(\mathrm{kWh})$. To instead consider power units $(\mathrm{kW})$, all profiles must be multiplied by 2 (since the time step is 0.5 hours). This gives the average load over 30 minutes.

The following outlines the clustering algorithm applied to forecast EV uptake.

1) Firstly, we establish the percentage of households in the sample population that will adopt EVs and the number of years it will take (This is set to be 8 years).

2) Next, an initial random distribution of EV seeds is performed to simulate the first year of EV uptake.

3) Then, during the remaining years, EVs are assigned to households according to the score $s$ (refer to (2)).

4) The number of EV households (households that adopted an EV) increases linearly every year until the specified amount is attained.

5) Lastly, EV profiles are assigned to the EV households, where

EV household profile $=$ base load + EV profile

It is important to note that all 71 commercial properties in our data set never receive LCT load since our focus is LCT household uptake. As well, there is one feeder comprised of only commercial properties, thus this site is always given zero LCT load.

In 2015, OFGEM (a UK regulatory authority for gas and electricity markets) increased the period covered by a single price control review to 8 years [17]. As detailed investment decisions must be outlined in this time horizon, we also use 8 years in our simulations.

The score, $s$, assigned to eligible households is the percentage of PVs and EVs in its neighbourhood presently, where

$s=100\left(\frac{\text { Number of neighbours with an EV and /or PV }}{\text { Number of neighbours }+1}\right)$

This score is proportional to the probability of selection by a random number generator. Figure 3 illustrates this

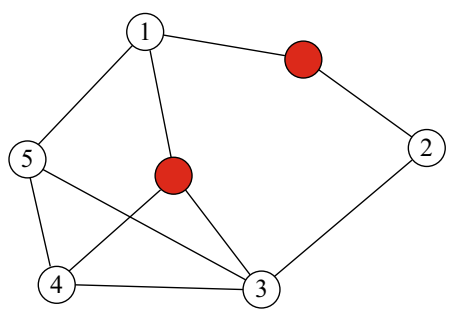

(a) Red circles signify EV allocation and the numbered circles correspond to eligible households (The connecting lines indicate neighbours)

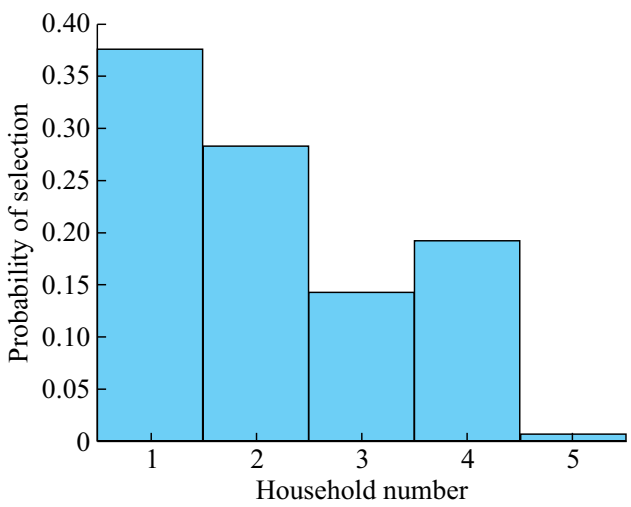

(b) Probability of EV assignment by the random number generator

Fig. 3 Illustration of selection process

selection process with a simplified network, comprising of red and numbered circles that represent EV and eligible households respectively, with their probability of EV assignment by the random number generator also shown. This figure suggests that household 1 is the most probable to acquire an $\mathrm{EV}$ due to observing 2 of its 3 neighbours with EVs.

Once a household is selected, they become an EV household for the remaining years of the simulation, with $s$ updated every year. Using $s$ to inform EV allocation leads to clusters of EVs forming around the initial seeds. Therefore, we are modelling the formation of green neighbourhoods due to social influence. This method is an adaptation of the algorithm proposed in [12], which was also applied to model EV uptake.

There is an assumed link between increased neighbourhood diversity and a heavily populated feeder. As a result, when transforming these larger sites into greener neighbourhoods, the impact from one EV household should be comparatively small. To account for this, $s$ depends upon the feeder population and therefore, the influence of one household on its neighbours is relative to the neighbourhood size.

Note that when a household is given an EV, the EV profile assigned to them is randomly selected from 79 possible profiles. If the baseload applied is representative 
of spring, summer or winter then the EV profile chosen will also correspond to spring, summer or winter respectively.

\section{Confidence bounds}

For some fixed model parameters, there are many feasible outcomes. This is due to the initial random distribution of seeds highlighting different neighbourhoods every model run. As well, one of 79 possible EV profiles are randomly assigned to households, causing further variation in the model result. Consequently, we aim to determine upper and lower bounds of the model response for a fixed set of parameters, which we label confidence bounds. These bounds will be calculated by undertaking 500 consecutive model runs and will therefore relate to the EV load variance, not the baseload. Since the clustering is based on neighbourhoods, which are defined by feeders, the bounds will be computed at each feeder.

The following details the method used to calculate confidence bounds.

1) Specify the model parameters, which are the uptake percentage and the number of years i.e. $30 \% \mathrm{EV}$ uptake ensures $\lceil 0.3 \times 1841\rceil$ properties receive an $\mathrm{EV}$ each simulation.

2) Complete 500 simulations.

3) After each simulation, record the aggregate result at the feeder. The 44 feeders are considered together so that $0 \%-100 \%$ of households along a particular feeder can receive an $\mathrm{EV}$ each simulation.

4) The aggregate data is then used to calculate $10 \%, 50 \%$ and $90 \%$ quantiles at the feeder. The feeder lower and upper bounds correspond to the $10 \%$ and $90 \%$ quantiles.

5) The quantile with the baseload subtracted represents the variation in EV load at the feeder. Then, dividing the quantiles by the number of households along the feeder, we can compare the 44 feeders and their EV loads.

In Fig. 4a, b, the results for feeders 15, 17, 39 and 40 are depicted when $30 \%$ uptake overall is assumed amongst the 44 feeders. The aggregate result at the feeder is displayed (including baseload), where the black dots represent the response from 500 model runs. The red, green and blue curves are the $10 \%, 50 \%$ and $90 \%$ quantiles respectively calculated from the black dots. The far right plots show the quantiles with the baseload subtracted, divided by the number of households along the feeder. Only the results corresponding to winter are given here, where the winter baseload and winter EV profiles discussed in Section 3 are used. From the far right plots of Fig. 4a, b, it is apparent that feeders with similar household numbers receive comparable EV loads, where feeders 15, 17, 39 and 40 have $25,27,82$ and 86 households respectively. Furthermore, less populated feeders have greater EV peaks, demonstrated by the blue curves. This can be attributed to increased neighbourhood diversity when the feeder population is larger and therefore, it is more difficult to influence your neighbours and form an EV majority. Also, it is evident that the spread between the $10 \%$ and $50 \%$ trends is far less notable for smaller feeders. Additionally, the red curve sits along the baseload in Fig. 4a. This suggests that less populated feeders do often avoid EV assignment.

Hence, similar to [11], multiple simulations are used to determine the impact of LCTs on a LV network. However, here the area examined is comparatively large, social influence is imposed and our focus is the load profile. Moreover, this methodology enables bounds for the expected load at each feeder/neighbourhood to be found, which are extremely informative measures for network planners.

Only the results for four of the 44 feeders considered by the model have been shown. Refer to the Appendix A for the depiction of the results across the entire network. In this paper, 30\% EV uptake has been selected. However, this is a model parameter that can be varied. Furthermore, the network examined here with our approach covers a fixed area, although, the analysis can be easily adapted to study networks of a greater or smaller size.

The number of simulations conducted, $n$, to calculate the bounds shown was $n=500$. Choosing $n=500$ was believed appropriate since the variance was captured but the computation time was minimised. This was deduced by comparing the confidence bounds at various feeders when $n=200, n=500$ and $n=1000$. These results revealed only minor differences between the quantiles for $n=500$ and $n=1000$. Therefore, considering the additional time required to undertake 1000 simulations, it was determined unnecessary to perform this many runs and setting $n=500$ was optimal for a network of this size.

These particular four feeders were chosen for closer analysis because feeders 15 and 17 have approximately the same number of households, similarly for feeders 39 and 40. As well, household numbers at feeders 15 and 17 are less than the average feeder population (which is 40 households), whereas they are greater at feeders 39 and 40, and therefore, two extreme cases are studied. Furthermore, the distribution of council tax bands (see Section 5) amongst these feeders varies considerably, which becomes important in Section 5.

The simulations and confidence bounds calculations were performed using MATLAB on a standard workstation with Intel i5 processor on $2.50 \mathrm{GHz}$ with $8 \mathrm{~GB}$ RAM. The computation time required to conduct 500 consecutive 


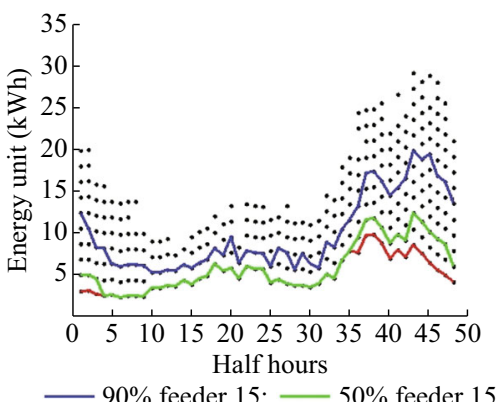

$-10 \%$ feeder 15

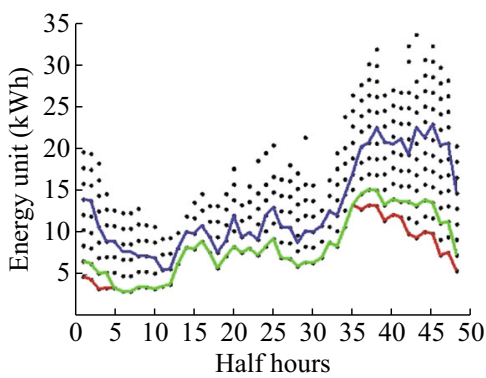

$-90 \%$ feeder $17 ;-50 \%$ feeder 17

$-10 \%$ feeder 17

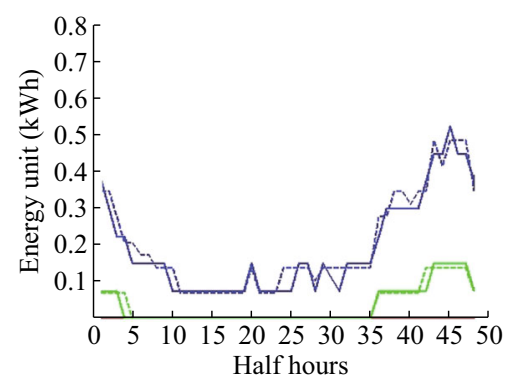

$-90 \%$ feeder $15 ;-50 \%$ feeder 15

$-10 \%$ feeder 15 ; ---- $90 \%$ feeder 17

$50 \%$ feeder 17 ; ---- $10 \%$ feeder 17

(a) Result of 500 simulations with $30 \% \mathrm{EV}$ winter uptake (random seed allocation)

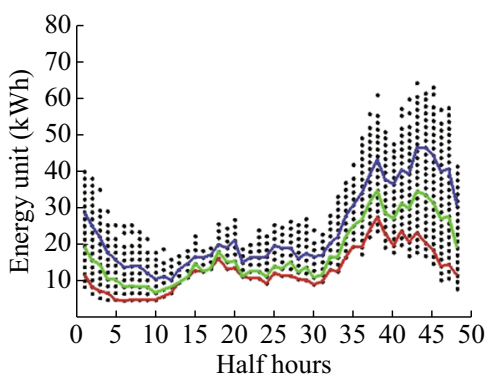

$-90 \%$ feeder $39 ;-50 \%$ feeder 39

$-10 \%$ feeder 39

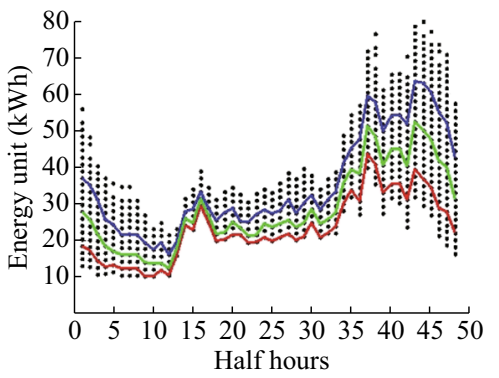

$-90 \%$ feeder $40 ;-50 \%$ feeder 40 $-10 \%$ feeder 40

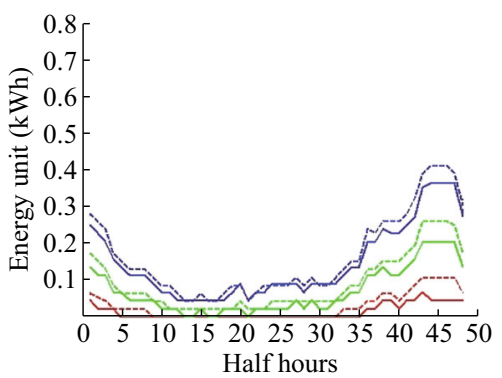

$-90 \%$ feeder $39 ;-50 \%$ feeder 39 $-10 \%$ feeder $39 ;-----90 \%$ feeder 40 -..- $50 \%$ feeder $40 ;$

(b) Result of 500 simulations with $30 \% \mathrm{EV}$ winter uptake (random seed allocation)

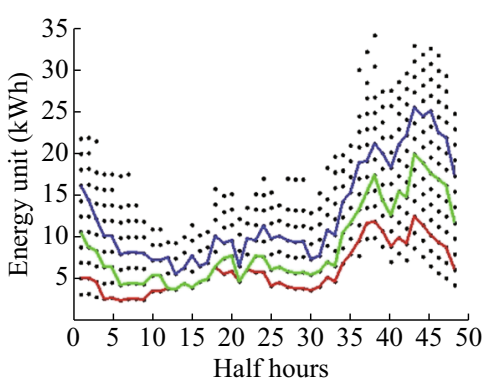

$-90 \%$ feeder $15 ;-50 \%$ feeder 15 $-10 \%$ feeder 15

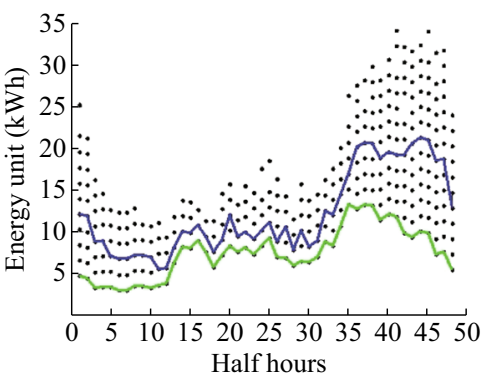

- $90 \%$ feeder $17 ;-50 \%$ feeder 17 $-10 \%$ feeder 17

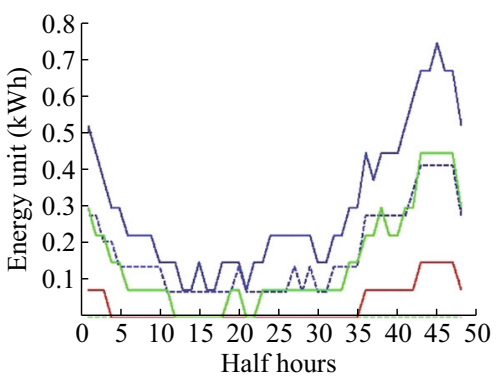

$-90 \%$ feeder $15 ;-50 \%$ feeder 15 $-10 \%$ feeder $15 ;----90 \%$ feeder 17 -- $50 \%$ feeder 17 ; ---- $10 \%$ feeder 17

(c) Result of 500 simulations with $30 \%$ EV winter uptake (seed allocation informed by CTB)

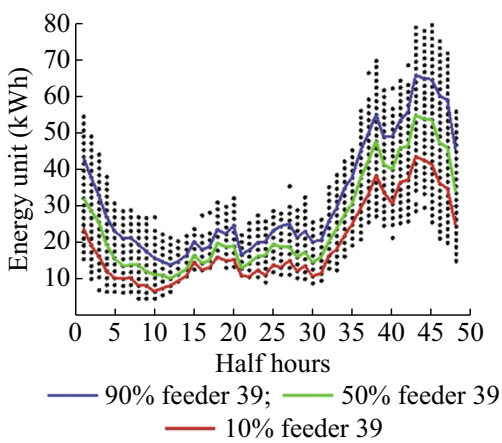

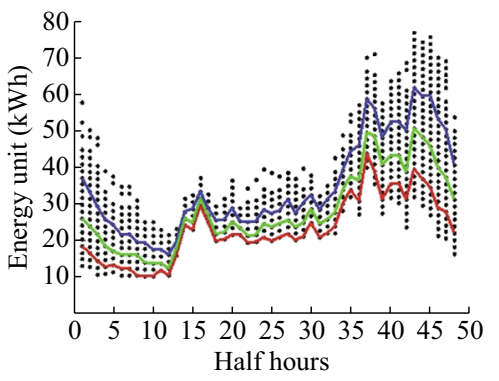

$90 \%$ feeder $40 ;-50 \%$ feeder 40

$-10 \%$ feeder 40

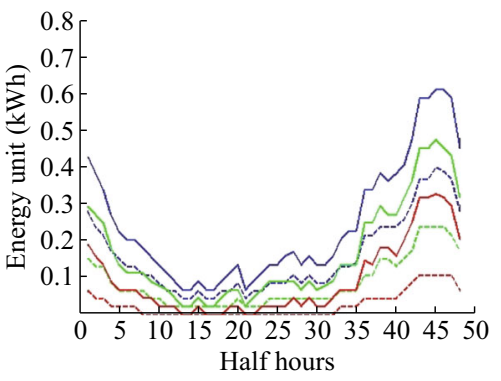

$-90 \%$ feeder $39 ;-50 \%$ feeder 39

$-10 \%$ feeder $39 ;----90 \%$ feeder 40

$50 \%$ feeder 40 ; ---- $10 \%$ feeder 40

(d) Result of 500 simulations with $30 \% \mathrm{EV}$ winter uptake (seed allocation informed by CTB)

Fig. 4 Simulation results 
model runs with this network (44 feeders consisting of 1841 properties) was approximately 15 minutes.

\section{Adding socio-demographic information}

The simulations performed in Section 4 randomly allocated EV seeds. Next, we aim to improve our model by introducing council tax band (CTB) information to instead inform seed distribution. Council tax is a local tax on domestic properties in England, Scotland and Wales, introduced in 1993. Each property is assigned one of eight bands ( $A$ to $H$ ) based on the property's capital value [18], where $A$ corresponds to the smallest value and $H$ to the highest. Here, it is assumed that larger homes have higher CTBs. We note here that other socio-demographic information can be used if available. We use CTB as it is publicly available [19] and it allows us to identify neighbourhoods that have a higher percentage of larger properties.

A survey of Californian EV owners [20] revealed that generally they owned and lived in single family dwellings that had parking and space to install a charging point. They also had higher incomes, which typically relates to living in larger homes. Furthermore, present EV owners commonly had a PV installed at their property. Also acknowledged was that neighbour influence was an important factor in EV adoption since clusters of EV households had formed in California. This study therefore supports initialising the seeds guided by CTB information and then imposing neighbour influence to determine the growth of $\mathrm{EV}$ ownership.

The model is now adapted to firstly favour PV properties and larger households, signified by higher CTBs. To implement this, we assign to every household an initial score, $s_{i}$, such that:

$s_{i}=\frac{100 C T B_{h h}^{j}}{8^{j}}$

where $C T B_{h h}=1,2, \ldots, 8$ when the household's CTB is $A, B, \ldots, H$ respectively and $j$ is some positive integer. As well, PV (resp. commercial) properties are given the score $s_{i}=100$ (resp. $s_{i}=0$ ). The score is proportional to the likelihood of selection by a random number generator. This relationship is consistent with that portrayed in Fig. 3. It should be noted that $s_{i}$ is only used during the first year when seeds are allocated, then $s$ as given by (2), applies for the remaining years.

Choosing $j$ determines how dependent seed assignment is on the CTB information, where CTB influence increases with $j$. Moreover, higher values of $j$ will result in prominent clusters developing in neighbourhoods with large households. Here we set $j=4$.
The last column of Table 1 details the spread of CTBs within our sample population of 1841 properties. As well, in Table 1 a comparison is given of 100 seeds that are selected using CTB information, with $j=1,2,3,4$, where the distribution of CTBs for the 100 nominated households is displayed. We propose that by setting $j=4$, the subsequent initial EV population reflects the survey findings [20], since approximately $70 \%$ of seeds now have a CTB greater than $C$ ( $A-C$ typically represents small dwellings). Note that $j$ is a model parameter that can be varied.

Confidence bounds can be used to measure the effect of changing our model assumptions. Here, we analyse the influence of using CTB to inform the initial seed distribution, instead of random initial distribution. The winter results for feeders 15, 17, 39 and 40 are shown in Fig. 4c, d. It is evident that feeders with about the same sized populations are no longer given a similar EV load. The upper bounds depicted along Fig. 4c reveal that feeder 15 receives a significantly larger load than feeder 17 . This is due to $60 \%$ of properties along feeder 15 having a CTB greater than $D$, whereas for feeder 17 it is $0 \%$. Similarly, Fig. $4 \mathrm{~d}$ suggests that feeder 39 has been assigned a greater EV load compared to feeder 40 , which is due to $54 \%$ of households along feeder 39 having a CTB of more than $D$, when feeder 40 has $0 \%$. Furthermore, of these four feeders, feeder 15 overall has the largest EV peak, which is a result of both feeder size and its households' CTBs. In Fig. 4a, c: feeder 15 has 25 households and the number of households with CTB $>D=60 \%$; panels a and c: feeder 17 has 27 households and the number of households with CTB $>D=0 \%$; panels $\mathrm{b}$ and d: feeder 39 has 82 households and the number of households with CTB $>D=54 \%$; panels $\mathrm{b}$ and d: feeder 40 has 86 households and the number of households with CTB $>D=0 \%$.

Now that socio-demographic data has been incorporated, higher potential peaks are exhibited at certain feeders than previously predicted. This modelling suggests that

Table 1 CTB Distributions

\begin{tabular}{lccccr}
\hline CTB & $j=1$ & $j=2$ & $j=3$ & $j=4$ & All \\
\hline 0 & 0 & 0 & 0 & 0 & 71 \\
A (1) & 0 & 0 & 0 & 0 & 1 \\
B (2) & 2 & 2 & 2 & 0 & 103 \\
C (3) & 59 & 47 & 46 & 31 & 1135 \\
D (4) & 25 & 32 & 24 & 29 & 373 \\
E (5) & 5 & 9 & 14 & 23 & 107 \\
F (6) & 8 & 5 & 8 & 8 & 37 \\
G (7) & 1 & 5 & 4 & 7 & 12 \\
H (8) & 0 & 0 & 2 & 2 & 2 \\
\hline
\end{tabular}




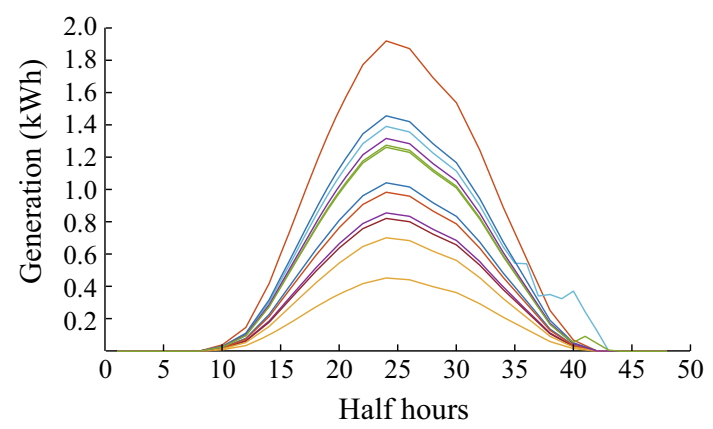

Fig. 5 Summer PV generation profiles

these feeders are likely sites for future network issues. Refer to the Appendix A for an overview of the results at all 44 feeders.

\section{Combination of electric vehicles and photovoltaics}

The additional impact of PV adoption on the electricity network is now examined by adapting our model to also consider PV uptake.

As part of the New Thames Valley Vision project, surplus generation and solar radiation data was recorded at 12 households that had PVs installed. PV daily generation profiles were then created, defined every half hour, by assuming that PV generation is proportional to solar radiation. It should be noted that other influences, such as ambient and surface temperatures, would also contribute to PV generation, but we ignore these to simplify our model. Thus, for our analysis only solar radiation is used. As a result, we obtained three sets of 12 PV daily generation profiles that were representative of spring, summer and winter generation. For this investigation, only the summer baseload and summer PV profiles are applied to simulate uptake. In Fig. 5, the 12 summer PV daily generation profiles are shown. These profiles are scaled so that the maximum generation is $1.9 \mathrm{kWh}$ to comply with UK standards. When a household is given a PV, one of the 12 possible profiles are randomly selected and then subtracted from their baseload i.e. a household with an EV and a PV is assigned the profile:

$$
\begin{aligned}
E V+P V \text { household profile }= & \text { baseload }+E V \text { profile } \\
& -P V \text { profile }
\end{aligned}
$$

The confidence bounds discussed in Section 4 are now used to quantify the effect of both EV and PV adoption by our sample population. Simulations for 30\% EV and 30\% $\mathrm{PV}$ uptake are conducted (the uptake percentage chosen is a model parameter, which can be modified). Firstly, the clustering algorithm outlined in Section 3 is applied, where the initial seed is randomly distributed. Then, the seed allocation is guided by CTB. There are now two scores assigned to eligible households. These are $s_{E V}$ and $s_{P V}$, where both are defined using (2) and updated every year. A household's likelihood for EV (resp. PV) selection by a random number generator is proportional to $s_{E V}$ (resp. $s_{P V}$ ). The dependence of selection on these scores is demonstrated by Fig. 3 .

Again the 71 commercial properties within our data set do not receive a LCT. Also, we ensure that the 7 households with PVs installed already are not allocated an additional PV.

In Fig. 6a, c: feeder 15 has 25 households and the number of households with CTB $>D=60 \%$; panels a and c: feeder 17 has 27 households and the number of households with CTB $>D=0 \%$; panels b and d: feeder 39 has 82 households and the number of households with CTB $>D=54 \%$; panels $\mathrm{b}$ and d: feeder 40 has 86 households and the number of households with CTB $>D=0 \%$.

In Fig. 6a, b, the results for feeders 15, 17, 39 and 40 are displayed. Here, the initial seeds have been randomly allocated, where EV and PV seeds are distributed separately. The quantiles depicted reveal significant troughs (red curve) develop during the day and large peaks (blue curve) form at night. Furthermore, it is evident that feeders of a similar size again receive comparable EV/PV loads, where the red trough and blue peak are more prominent for smaller feeders, suggesting increased variability at these feeders.

Next, in Fig. 6c, d, the results for feeders 15, 17, 39 and 40 are given, where now the seed distribution is informed by CTB information. The allocation of EV and PV seeds are again separate. Consistent with previous findings, due to introducing CTB, feeders 15 and 39 have greater extremes. Interestingly, these values are roughly the same for feeders 15 and 39, and hence, independent of feeder size. This is a result of now modelling two technologies, which amplifies the clustering effect. Although, the result variability is more pronounced for the smaller feeders, indicated by the spread of the quantiles. As a result of using CTBs, the minimum and maximum loads obtained at feeders 15 and 39 are now larger than initially estimated (see Fig. 6a, b).

Lastly, simulations of 30\% EV and 30\% PV uptake with CTB information are again performed, except now we ensure that all households which receive an EV with our clustering algorithm are also given a PV. The results are actually extremely similar to those depicted in Fig. 6c, d. The most significant difference observed is at feeder 17 and is shown in Fig. 7. This is expected as our clustering method already promotes the growth of $\mathrm{EV}+\mathrm{PV}$ groupings. 


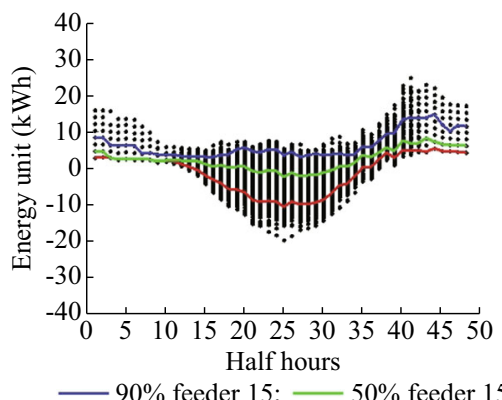

$-90 \%$ feeder 15 ; $-50 \%$ feeder 15 $-10 \%$ feeder 15

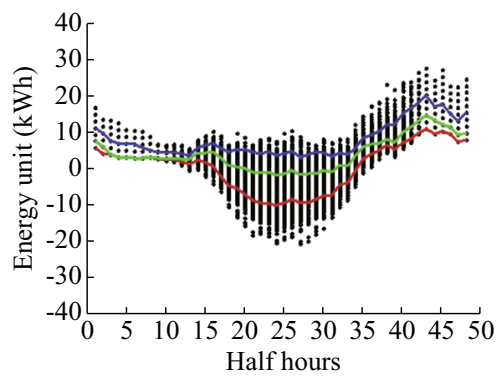

$-90 \%$ feeder 17 ; $-50 \%$ feeder 17

$-10 \%$ feeder 17

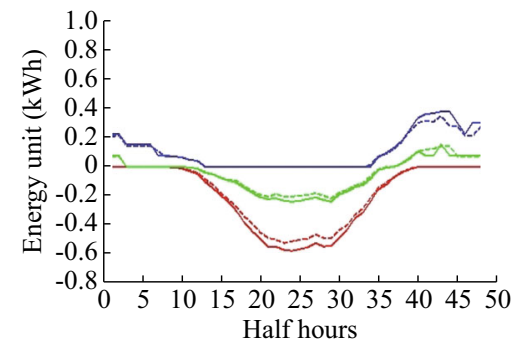

$-90 \%$ feeder $15 ;-50 \%$ feeder 15 $-10 \%$ feeder 15 ; ---- $90 \%$ feeder 17 --- $50 \%$ feeder 17 ; ---- $10 \%$ feeder 17

(a) Result of 500 simulations with $30 \% \mathrm{EV}$ uptake and 30\% PV uptake summer (random seed allocation)

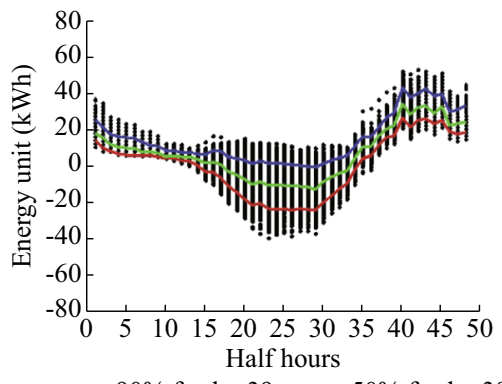

$-90 \%$ feeder $39 ;-50 \%$ feeder 39 $-10 \%$ feeder 39

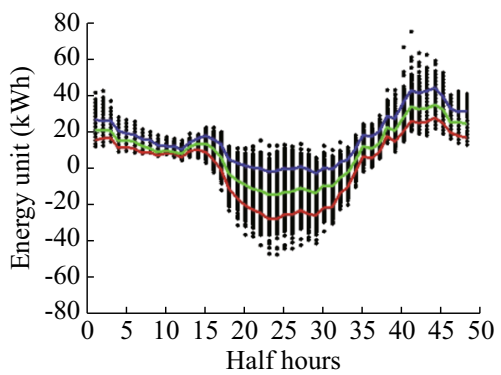

$-90 \%$ feeder $40 ;-50 \%$ feeder 40

$-10 \%$ feeder 40

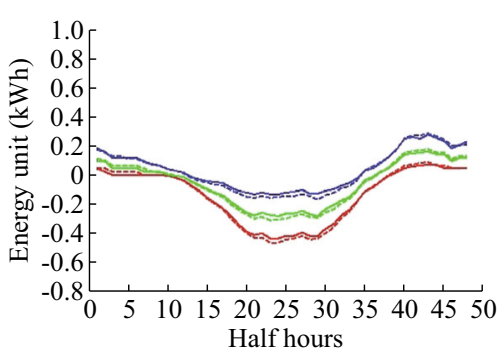

$-90 \%$ feeder $39 ;-50 \%$ feeder 39

$-10 \%$ feeder $39 ;-----90 \%$ feeder 40

$50 \%$ feeder 40 ; ---- $10 \%$ feeder 40

(b) Result of 500 simulations with $30 \% \mathrm{EV}$ uptake and 30\% PV uptake summer (random seed allocation)

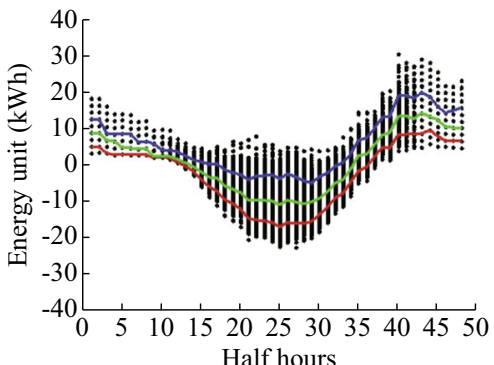

$-90 \%$ feeder $15 ;-50 \%$ feeder 15 $-10 \%$ feeder 15

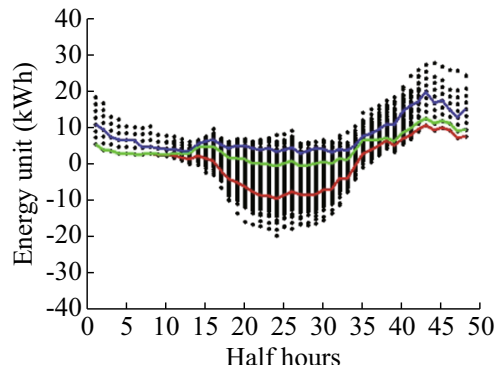

$-90 \%$ feeder 17 ; $-50 \%$ feeder 17 $-10 \%$ feeder 17

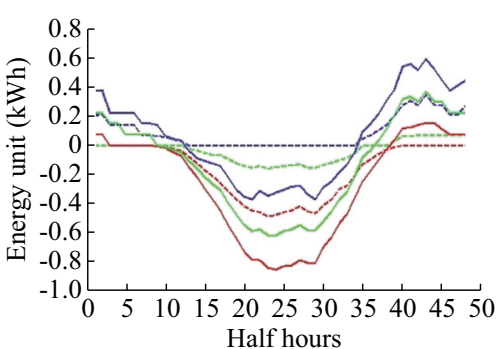

$-90 \%$ feeder $15 ;-50 \%$ feeder 15

$-10 \%$ feeder 15 ; ---- $90 \%$ feeder 17 $50 \%$ feeder 17 ; ---- $10 \%$ feeder 17

(c) Result of 500 simulations with 30\% EV uptake and 30\% PV uptake summer (seed allocation informed by CTB)

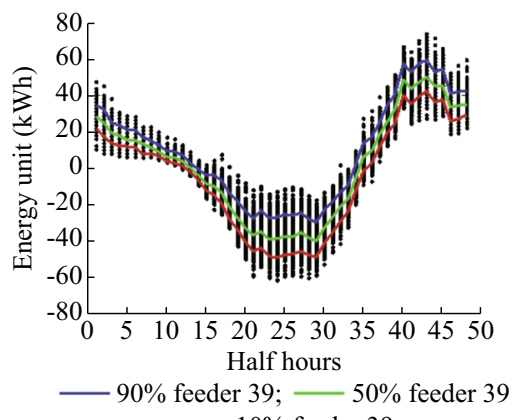

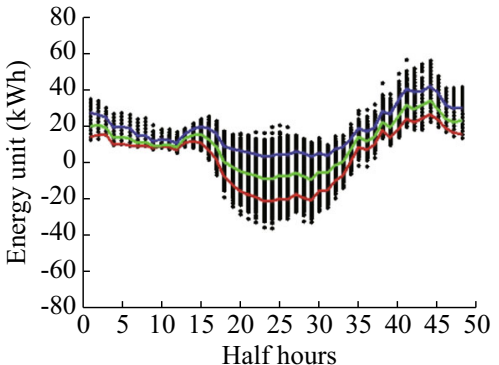

$-90 \%$ feeder $40 ;-50 \%$ feeder 40 $-10 \%$ feeder 40

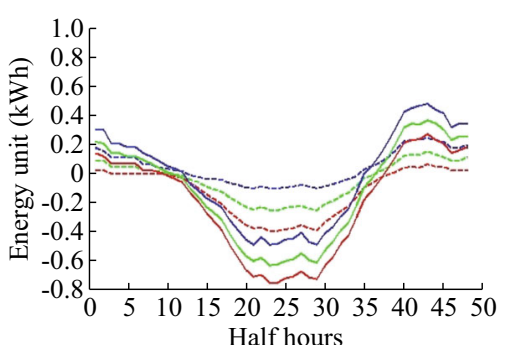

- $90 \%$ feeder $39 ;-50 \%$ feeder 39 $-10 \%$ feeder $39 ;$----- $90 \%$ feeder 40 -.-. $50 \%$ feeder 40 ; ---- $10 \%$ feeder 40

(d) Result of 500 simulations with 30\% EV uptake and 30\% PV uptake summer (seed allocation informed by CTB)

Fig. 6 Simulation results 

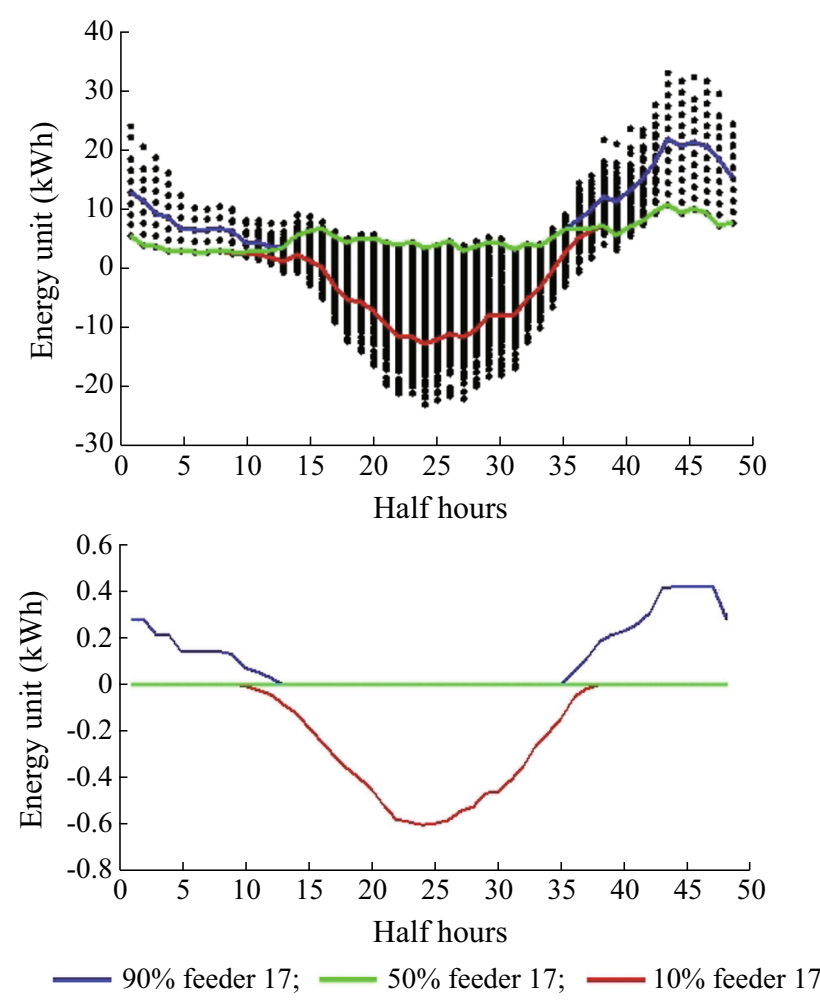

Fig. 7 Result of 500 simulations with 30\% EV uptake and 30\% PV uptake summer (seed allocation informed by CTB, every household that obtained an EV were also given a PV)

Modelling EV and PV uptake reveals significant nighttime peaks and daytime troughs, which enlarge at some feeders when the CTB information is applied. These results can inform network operators about the need for reinforcing some of the feeders so to cater for the load increases at night and voltage rises during the day, which is due to $\mathrm{EV}$ charging and PV generation respectively. Refer to the Appendix A where an overview of the results at the 44 feeders is given.

Analysing load profiles is a straightforward method to assess the impact of LCTs and to highlight potential sites that require further attention. However, other factors such as thermal and voltage issues must also be considered to fully understand the LCT impact. To explore these issues, the feeder upper and lower bounds obtained through our modelling are used by a network operator as input to a voltage and thermal constraints simulator.

\section{Conclusion}

An agent based model was outlined that considered social factors to predict the uptake of low-carbon technologies. The data used was taken from real-life, with real substation and feeder assignment. This allowed us to sort the 1841 properties into 44 realistic neighbourhoods. Then neighbour influence was imposed to determine uptake. The model also applied sets of EV and PV profiles that were representative of spring, summer and winter usage. To assess the model response, a probabilistic approach was proposed that provided feeder confidence bounds, which were an upper and lower limit for the expected load at every neighbourhood. These were a result of 500 consecutive simulations and therefore, the bounds measured the variation in LCT load. Next, another aspect of social influence was introduced with socio-demographic information also guiding LCT selection. More specifically, we ensured that bigger households were more likely to acquire a LCT. Confidence bounds were then utilised to quantify the effect of implementing this change. In particular, the potential peaks/troughs at select feeders were amplified as these neighbourhoods comprised of clusters of larger homes. The modelling undertaken focussed on EV adoption and then the combination of EV and PV uptake. To investigate these different scenarios and their possible model outcomes, computing confidence bounds was extremely effective. Moreover, the upper bound can also be used to determine the available headroom at each feeder for some specified uptake percentage. Identifying headroom is essential for network planning since negative headroom indicates transmission is greater than the maximum available power, causing issues for the electricity provider. Hence, subsequent to the upper bound calculation, certain feeders can be highlighted as likely sites for network malfunction when subjected to LCT demand. Furthermore, when analysing PV uptake as well, the lower bound becomes an equally important measure since negative power at the feeder level is also problematic for the electricity provider. As well, future voltage and thermal issues can be examined with these bounds if used as input to a voltage and thermal constraints simulator. Confidence bounds therefore will be an important tool to inform new policies and planning so that the future impact of LCTs on the LV network can be mitigated.

Acknowledgements This work was supported by Scottish and Southern Electricity Networks through the New Thames Valley Vision Project (SSET203 New Thames Valley Vision), and funded by the Low Carbon Network Fund established by Ofgem.

Open Access This article is distributed under the terms of the Creative Commons Attribution 4.0 International License (http:// creativecommons.org/licenses/by/4.0/), which permits unrestricted use, distribution, and reproduction in any medium, provided you give appropriate credit to the original author(s) and the source, provide a link to the Creative Commons license, and indicate if changes were made. 


\section{Appendix A}

The confidence bounds results for the studies:

1) $30 \% \mathrm{EV}$ uptake winter with

a) seeds randomly distributed;

b) seed distribution guided by CTB information.

2) $30 \% \mathrm{EV}$ and $30 \% \mathrm{PV}$ uptake summer with

a) seeds randomly distributed;

b) seed distribution guided by CTB information.

are summarised in Fig. 8 for all 44 feeders. Note that feeder 1 is comprised of only commercial properties, so this

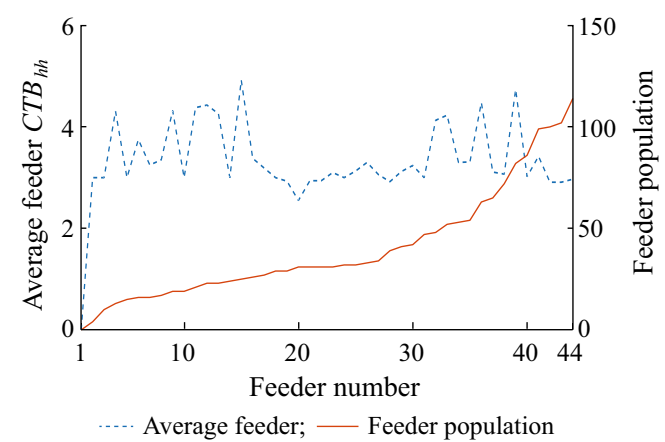

(a) The average $C T B_{h h}$ at each feeder (blue-dashed) and the variation in the feeder household population (red)

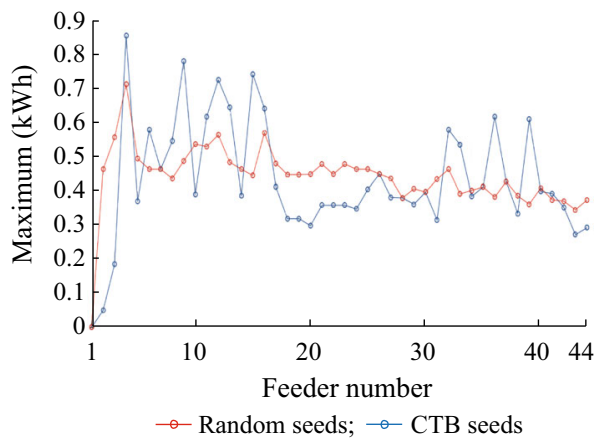

(b) The peak value of the feeder $90 \%$ quantile, divided by the number of households along this feeder (study a)

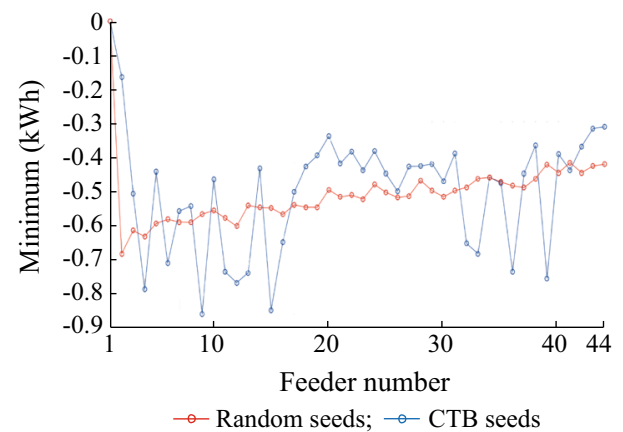

(c) The minimum value of the feeder $10 \%$ quantile, divided by the number of households along this feeder (study b)

Fig. 8 Overview of results for all 44 feeders feeder does not receive any LCTs. Panel a depicts each feeder's average $C T B_{h h}$ (blue-dashed) and household population (red). Panel $\mathrm{b}$ relates to study $a$, where the maximum value of the $90 \% \mathrm{EV}$ feeder quantile (the baseload subtracted), divided by the number of households along the feeder, is shown. The red and blue curve correspond to $a(i)$ and $a(i i)$ respectively. Panel c displays the results for study $b$, with the minimum value of the $10 \%$ EV/PV feeder quantile (the baseload subtracted), divided by the number of households along the feeder, given. The red and blue trend are linked with $b(i)$ and $b(i i)$ respectively. There is an evident correlation between the feeder population and the red curves associated with $a(i)$ and $b(i)$. This behaviour was discussed in Section 4, where less populated feeders received larger LCT loads. When the CTB data is introduced, certain feeders attain greater extreme values, whilst at other feeders the load magnitude is reduced. This is demonstrated by the blue curve along the bottom two panels, which overall follows the top panel blue trend. This is expected since when CTBs are applied, the clustering algorithm favours feeders that have a higher proportion of larger properties. The feeders that receive amplified minimum and maximum values are especially vulnerable, therefore further analysis and possibly reinforcement at these sites are needed.

\section{References}

[1] Department of Energy \& Climate Change (2014) UK energy statistics: statistical press release. https://www.gov.uk/government/ news/uk-energy-statistics-statistical-press-release-september-2014

[2] National Grid (2015) Electricity ten year statement. National Grid, London. http://www2.nationalgrid.com/UK/Industryinformation/Future-of-Energy/Electricity-ten-year-statement/

[3] Bilton M, Chike NE, Woolf $M$ et al (2014) Impact of low voltage-connected low carbon technologies on network utilisation. Report B4. Imperial College London, London, UK

[4] Bossmann T, Staffell I (2015) The shape of future electricity demand: exploring load curves in 2050s Germany and Britain. Energy 90(2):1317-1333

[5] ElNozahy MS, Salama MMA (2013) Technical impacts of gridconnected photovoltaic systems on electrical networks: a review. J Renew Sustain Energy 5(3):116-120

[6] Yong JY, Ramachandaramurthy VK, Tan KM et al (2015) A review on the state-of-the-art technologies of electric vehicle, its impacts and prospects. Renew Sustain Energy Rev 49:365-385

[7] Neaimeh M, Wardle R, Jenkins AM et al (2015) A probabilistic approach to combining smart meter and electric vehicle charging data to investigate distribution network impacts. Appl Energy 157:688-698

[8] Liu MM, McNamara P, Shorten R et al (2015) Residential electrical vehicle charging strategies: the good, the bad and the ugly. J Mod Power Syst Clean Energy 3(2):190-202. doi:10. 1007/s40565-015-0122-2

[9] Watson JD, Watson NR, Santos-Martin D et al (2016) Impact of solar photovoltaics on the low-voltage distribution network in New Zealand. IET Gener Transm Distrib 10(1):1-9 
[10] van der Kam M, van Sark W (2015) Smart charging of electric vehicles with photovoltaic power and vehicle-to-grid technology in a microgrid; a case study. Appl Energy 152:20-30

[11] Navarro-Espinosa A, Ochoa LF (2016) Probabilistic impact assessment of low carbon technologies in LV distribution systems. IEEE Trans Power Syst 31(3):2192-2203

[12] Poghosyan A, Greetham DV, Haben S et al (2015) Long term individual load forecast under different electrical vehicles uptake scenarios. Appl Energy 157:699-709

[13] Mullan J, Harries D, Braunl T et al (2011) Modelling the impacts of electric vehicle recharging on the Western Australian electricity supply system. Energy Policy 39(7):4349-4359

[14] Adepetu A, Keshav S, Arya V (2016) An agent-based electric vehicle ecosystem model: San Francisco case study. Transp Policy 46:109-122

[15] Giasemidis G, Haben S, Lee T et al (2016) A genetic algorithm approach for modelling low voltage network demands. https:// arxiv.org/abs/1612.06833

[16] EA Technology Limited (2016) My electric avenue

[17] RIIO-ED1 price control. https://www.ofgem.gov.uk/electricity/ distribution-networks/network-price-controls/riio-ed1-price-control
[18] Understand how council tax bands are assessed. http://www. lancaster.gov.uk/council-tax/council-tax-bands/

[19] Council Tax. https://www.gov.uk/council-tax/working-out-yourcouncil-tax

[20] California Center for Sustainable Energy (2013) California plugin electric vehicle driver survey results

Laura HATTAM is a Postdoctoral Research Assistant within the Centre for the Mathematics of Human Behaviour at the University of Reading. Her research interests include dynamical systems theory and its applications, and mathematical modelling.

Danica Vukadinovic GREETHAM is Lecturer in Mathematics and Director of the Centre for the Mathematics of Human Behaviour at the University of Reading. Her main expertise is in algorithmic graph theory and network modelling and analysis. Her research interests include mathematical modeling of low voltage networks. 